asthmatic fibrocytes (1-EBIO-dependent current at $+40 \mathrm{mV} 969 \pm 216$ $\mathrm{pA} ; \mathrm{n}=17$ cells) compared to healthy fibrocytes (1-EBIO-induced current $506 \pm 94 \mathrm{pA} ; \mathrm{n}=30$ cells; $\mathrm{p}=0.029) . \mathrm{K}_{\mathrm{Ca}} 3.1$ currents were blocked by the selective $\mathrm{K}_{\mathrm{Ca}} 3.1$ blocker TRAM-34. The migration of differentiated fibrocytes induced by airway smooth muscle supernatant was inhibited by $71.1 \pm 6.9 \%$ by TRAM-34 $200 \mathrm{nM}(\mathrm{p}<0.0001)$. The CXCL12-dependent migration of fibrocyte progenitors within a freshly isolated PBMC population was reduced by $70.9 \pm 7.9 \%$ by TRAM-34 ( $p=0.003)$.

Conclusions The $\mathrm{K}^{+}$channel $\mathrm{K}_{\mathrm{Ca}} 3.1$ is expressed in human fibrocytes, and plays a key role in their migration. $\mathrm{K}_{\mathrm{Ca}} 3.1$ blockers may therefore offer a novel approach to the treatment of airway wall remodelling in asthma, and parenchymal fibrosis in idiopathic pulmonary fibrosis.

\section{S46 ACTIVATION OF TGF- $\beta$ BY AIRWAY SMOOTH MUSCLE CELLS VIA THE $\alpha V \beta 5$ INTEGRIN IN ASTHMATIC AIRWAY REMODELLING}

doi:10.1136/thx.2010.150912.46

A L Tatler, A E John, L Jolly, A J Knox, L Pang, G Jenkins. University of Nottingham, Nottingham, UK

Airway remodelling is a common feature of severe asthma. Transforming growth factor- $\beta$ (TGF- $\beta$ ) is a pro-fibrotic, pleiotropic cytokine implicated in airway remodelling. TGF- $\beta$ is sequestered in the extracellular matrix as a latent complex and requires activation to function. Lysophosphatidic acid (LPA) causes TGF- $\beta$ activation in airway epithelial cells. The study aims were to investigate the effect of LPA on TGF- $\beta$ activation by ASM cells in asthma. TGF- $\beta$ activation was assessed by a reporter cell co-culture assay, by determining expression of the TGF- $\beta$-inducible gene plasminogen activator inhibitor-1 (PAI1) and by detecting the nuclear translocation of Smad proteins. The effect of LPA on TGF- $\beta$ activation in asthma was investigated by comparing the responses of ASM cells from non-asthmatic $(n=3)$ and asthmatic $(n=3)$ donors. TGFb activation was also assessed using a chronic ovalbumin model of airway remodelling in mice. LPA induced a time, and concentration, dependent increase in TGF- $\beta$ activation by ASM cells that was abrogated by an integrin $\alpha \mathrm{V} \beta 5$ antibody. An inhibitor of cytoskeletal reorganisation inhibited the effects of LPA. Furthermore, the $\beta 2$ agonist formoterol inhibited LPA-induced PAI1 expression. Primary asthmatic ASM cells activated more TGF- $\beta$ via $\alpha \mathrm{V} \beta 5$ in response to LPA than control cells, however they did not express more $\alpha \mathrm{V} \beta 5$ on the cell surface. Phosphorylation of Smad2 and expression ofpai1 in the lungs was increased in a chronic ovalbumin model of asthmatic airway remodelling in mice. Furthermore, $\alpha \mathrm{V} \beta 5$ integrin staining and $\alpha$-smooth muscle actin staining in the ASM layer around the airways is increased in this model. Collectively, these data show that ASM cells can activate TGF- $\beta$ via the $\alpha \mathrm{V} \beta 5$ integrin and highlight a novel pathway of TGF- $\beta$ activation in ASM cells, which may be important in development of asthmatic airway remodelling.

\section{Inflammation in lung injury: the key mediators S47 RAGE-MEDIATED CYTOKINE RELEASE FROM LEUKOCYTES:}

doi:10.1136/thx.2010.150912.47

M Kaneshamoorthy, B C Creagh-Brown, A Burke-Gaffney. Unit of Critical Care, Respiratory Science, National Heart and Lung Institute Division, Faculty of Medicine, Imperial College, London, UK

Introduction Systemic inflammatory response syndrome (SIRS) is the physiological and biochemical changes that result from an
Pre versus post-op - IL-8

$n=3$

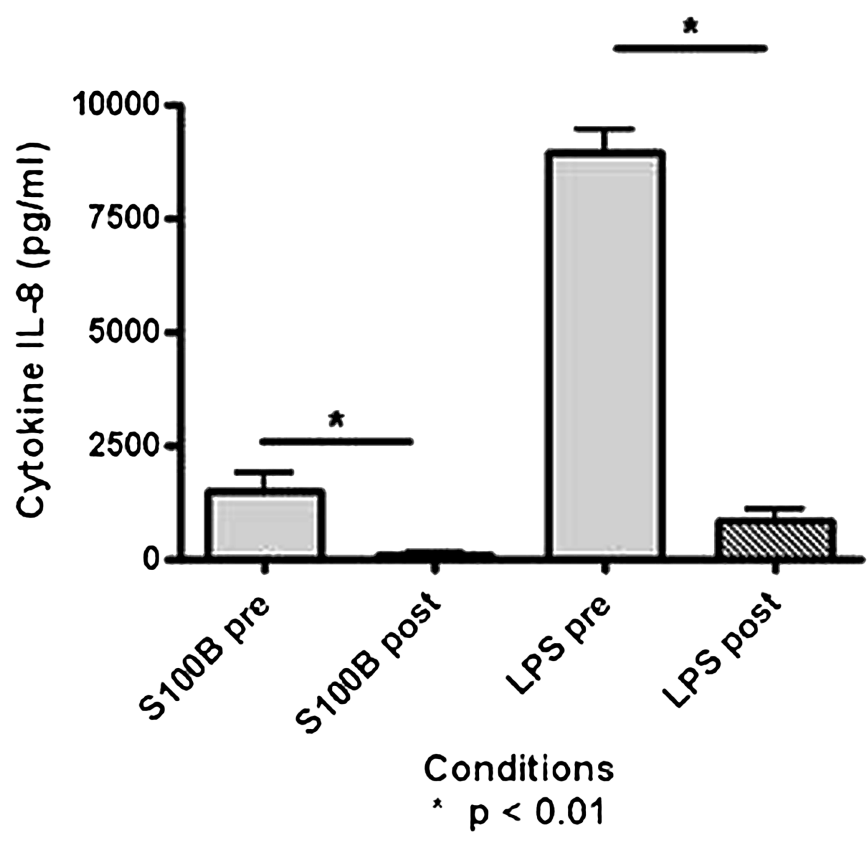

Pre versus post-op IL-10 $n=3$

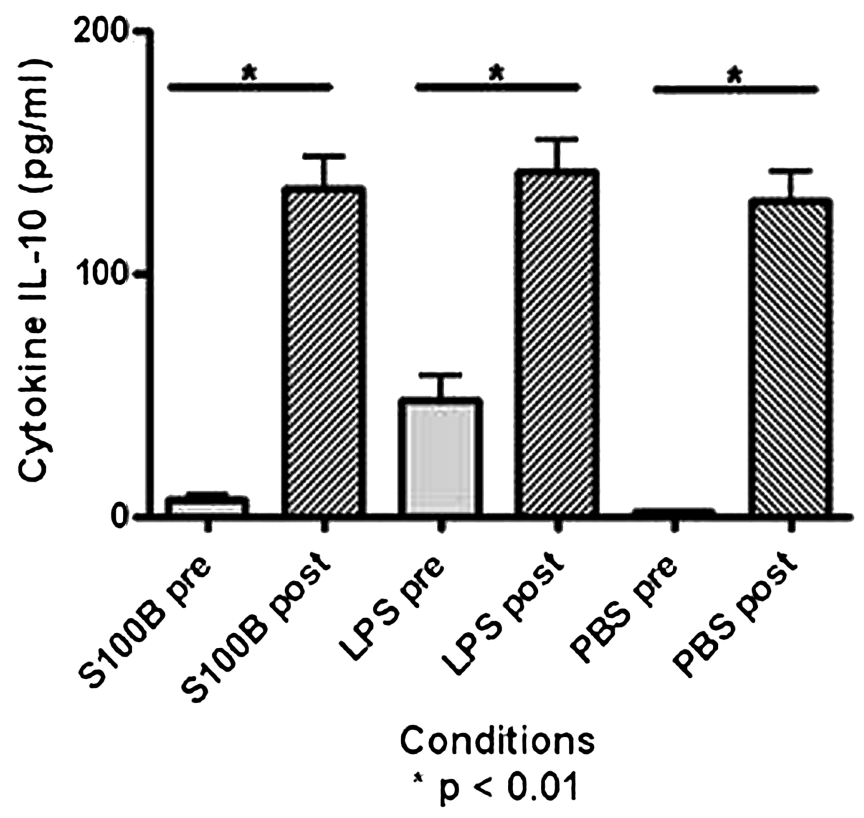

Abstract S47 Figure 1a and b Graph a shows the pre (grey) and post-bypass (striped) effects of the stimuli S100B and LPS in releasing the pro-inflammatory cytokine IL-8. The graph shows in the presence of S100B and LPS, IL-8 release is significantly reduced in post-bypass $(p<0.01)$, showing a reduced responsiveness to $S 100 B$ and LPS. Graph $b$ shows the pre and post-bypass effects of the stimuli S100B and LPS in releasing the IL-10. The graph shows that in the presence of S100B and LPS, all of the mean IL-10 release is significantly increased in post-bypass $(p<0.01)$. The mean cytokine release was calculated from nine patients done in duplicates. $p<0.01$ shows a significant difference between groups joined by a bar. PBS=Phosphate Buffered Saline; LPS=lipopolysaccharide. 
inflammation of the body, which commonly occurs in patients after cardiac surgery necessitating cardiopulmonary bypass (snCBP). To date, attempts at targeting inflammatory mediators associated with SIRS have been largely unsuccessful. One reason is there is a need to identify mediators involved in SIRS with a wider therapeutic window-one possibility is RAGE. RAGE is a transmembrane receptor, which acts as a pattern recognition receptor and induces inflammation. RAGE has multiple ligands, which include S100B and HMGB-1 proteins. RAGE ligands are raised in patients with SIRS but effects of RAGE ligands on cytokine release from whole blood, which could contribute to the onset of SIRS, is not well characterised. This study investigates the effects of S100B and HMGB-1 on cytokine release in whole blood (WB) pre and post-snCPB.

Methods Nine patients undergoing snCPB at the Royal Brompton Hospital were enrolled into the study. Blood was collected from patients pre- and post-snCPB. WB was incubated with PBS, LPS, S100B, or HMGB1 were added for $4 \mathrm{~h}$. Supernatants were collected and later assayed for IL-8 by ELISA (R\&D) and IL-1b, IL-6, IL-8, IL10, TNFa, IL-12p70 and IFN-g, by electrochemiluminescence multiplex analysis.

Results S100B and LPS caused significant release of IL-8 from WB acquired pre-op $(p<0.01)$. Significant release of IL-1b, IL-10, IL-6, IFN-g, TNFa and IL-12p70 was also detected. There was a significant reduction of the release of all cytokines, except IL-10, in the presence of S100B and LPS post-snCPB $(\mathrm{p}<0.01)$ compared with presnCPB release. IL-10 was significantly and similarly increased, irrespective of the experimental condition. HMGB-1 showed no significant cytokine release pre and post-snCPB.

Conclusion This study shows that S100B, like LPS, has pro-inflammatory activity in WB. Both stimuli had a hypo-responsive response post-snCPB. Current literature already describes the LPS response, but the response by S100B has not previously been reported. Therefore S100B, but not HMGB-1, could be a potential pharmaceutical target in reducing inflammation in $\mathrm{snCPB}$ patients with SIRS.

\section{S48 NGAL INHIBITS CYTOSKELETAL RE-ORGANISATION, MMP-2 PRODUCTION AND INVASION IN ALVEOLAR EPITHELIAL CELLS IN AN IN VITRO MODEL OF ACUTE LUNG INJURY}

doi:10.1136/thx.2010.150912.48

C M O'Kane, E Moran, D F McAuley. Centre for Infection and Immunity, Queen's University of Belfast, Belfast, UK

Introduction We have identified bronchoalveolar lavage NGAL (neutrophil gelatinase associated lipocalin, also known as lipocalin2) as a potential biomarker in acute lung injury (ALI). Rising NGAL concentrations over the first $72 \mathrm{~h}$ of illness, and higher absolute NGAL levels at day 3 predicted ICU survival. The biological mechanism by which NGAL is associated with improved survival is not known. Lipocalin-2 modulates the process of epithelial to mesenchymal transition (EMT) in breast cancer. We have demonstrated the typical morphological and proteomic changes and the invasive phenotype of EMT in alveolar epithelial cells in vitro in response to either ARDS BAL fluid or TNFalpha + TGFbeta, cytokines present in the lavage fluid of patients with ALI. We hypothesised that NGAL could alter EMT in vitro in alveolar epithelium.

Methods A549 cells were stimulated with TNFalpha + TGFbeta in the presence or absence of NGAL $(0.1-10 \mathrm{ng} / \mathrm{ml})$. Cells were lysed at $72 \mathrm{~h}$ and probed by western blot for E-cadherin, Zo-1, fibronectin and vimentin. Supernatants were analysed by gelatin zymography for MMP-2. The invasive capacity of the cells was assessed using BD Biocoat Matrigel Invasion Assay Chambers.

Results As previously described TNFalpha + TGFbeta caused loss of E-cadherin, Zo-1 and gain of vimentin and fibronectin in A549 cells, with an accompanying morphological change to spindle shaped cells, that secreted MMP-2 and invaded matrigel. Treatment with NGAL did not affect E-cadherin, Zo-1, fibronectin nor vimentin. However, treatment with NGAL abrogated the change in morphology, maintaining an epithelial-like cobblestone appearance (Abstract S48 Figure 1a). Additionally, NGAL inhibited the production of MMP-2 by these cells (from $1.8 \pm 0.3$ to $0.9 \pm 0.2$ relative gelatinolytic units, $p=0.04$ ) and reduced the capacity of the cells to migrate through basement membrane-type matrix (Abstract S48 Figure 1b).
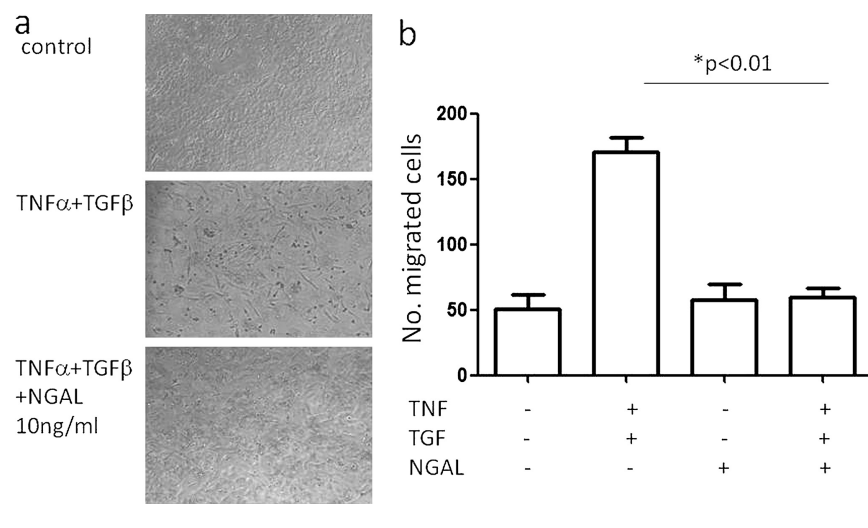

\section{Abstract S48 Figure 1}

Conclusion NGAL inhibited some but not all of typical changes of EMT in this in vitro model of ALI. The protective effect of NGAL in patients with ALI may be mediated by maintaining alveolar epithelial morphology, reducing their production of the basement membrane-destructive enzyme MMP-2, and reducing their capacity to migrate to the sub-epithelial interstitial space. Further work is required to assess this mechanism in vivo and to examine the effects of supplementary NGAL in ALI.

\section{S49 IS THE DEVELOPMENT OF ACUTE LUNG INJURY INFLUENCED BY INCREASED LEVELS OF IL17 AS A RESULT OF TREG/TH17 IMBALANCE?}

doi:10.1136/thx.2010.150912.49

${ }^{1} \mathrm{R}$ C A Dancer, 'V D'Souza, 'L Jeffery, ${ }^{1} \mathrm{D}$ Sansom, ${ }^{1} \mathrm{C}$ R Bassford, ${ }^{2} \mathrm{G}$ Perkins, 'D R Thickett. ' University of Birmingham, Birmingham, UK; ${ }^{2}$ University of Warwick, Warwick, UK

Introduction Vitamin D is known to have profound effects on the immune system. We have shown that vitamin $\mathrm{D}$ is lower in patients with Acute Lung Injury (ALI) than in healthy or at risk controls and that in patients at risk of ALI post oesophagectomy, low vitamin D levels are associated with increased post-operative systemic inflammatory response and alveolar epithelial dysfunction. Studies have shown that when $T$ cells are exposed to vitamin $\mathrm{D}$, expression of IL17 decreases and regulatory capacity increases. We hypothesised that vitamin $\mathrm{D}$ deficiency may play a role in development of Acute Lung Injury (ALI) via changes in the balance between regulatory $\mathrm{T}$ cells (Treg) and pro-inflammatory Th17 cells.

Methods Plasma levels of 25-OH Vitamin D (Tandem mass spectrometry) and 1,25-OH Vitamin D (ELISA)were measured in samples from patients with ALI. Normal T cells were exposed to BAL from patients with ALI with or without addition of exogenous vitamin $\mathrm{D}$ and determined frequencies of Treg and Th17 cells using flow cytometry.

Results All samples tested had insufficient plasma levels of $25-\mathrm{OH}$ vitamin $\mathrm{D}(<75 \mathrm{nmol} / \mathrm{l}$, median $14.1 \mathrm{nmol} / \mathrm{l}) .1,25$ vitamin $\mathrm{D}$ levels ranged from $<20$ to $176 \mathrm{pmol} / \mathrm{l}$ (reference range $43-144 \mathrm{pmol} / \mathrm{l}$ ) 1,25 vitamin D levels were significantly related to both ITU survival $(p=0.04)$ and survival at 90 days $(p=0.04)$. Our initial findings 